\title{
Diet and supplements as a supportive treatment for age-related macular degeneration
}

\author{
Jan 0strowski ${ }^{1}$, Karolina Kątska-0strowska', Wieslaw I. Gruszecki ${ }^{3}{ }^{3}$, Robert Rejdak $\oplus^{1}$ \\ ${ }^{1}$ Department of General Ophthalmology, Medical University of Lublin, Lublin, Poland \\ ${ }^{2}$ Department of Diagnostic and Microsurgery of Glaucoma, Medical University of Lublin, Lublin, Poland \\ ${ }^{3}$ Department of Biophysics, Institute of Physics, Maria Curie-Sklodowska University, Lublin, Poland
}

\begin{abstract}
Age-related macular degeneration (AMD) is one of the leading causes of blindness in developed countries. It is estimated that about 196 million people worldwide suffer from AMD. So far, despite many studies, no causative treatment that can be used large scale has been discovered. In order to slow down, the progression of the disease, a change of the modifiable risk factors such as diet and prophylaxis in the form of nutritional supplements are recommended. The purpose of this work is to present types of supplementation and draw attention to the proper diet containing products rich in substances such as lutein and zeaxanthin protecting the eye against the rapid progression of dry AMD.
\end{abstract}

KEY WORDS: AMD; dietary supplements; carotenoids; xanthophylls; lutein, zeaxanthin

Ophthalmol J 2020; Vol. 5, 101-106

\section{INTRODUCTION}

Age-related macular degeneration (AMD) is one of the leading causes of non-reversible visual impairment in humans over 50 years old and frequently increases in those after 65 years of age [1]. The prevalence of AMD is about 196 million people worldwide $[2,3]$. The disease affects the central part of retina called macula lutea and results in loss of central and sharp vision which is required for daily living activities as reading, driving and watching television.

Age-related macular degeneration can be classified as non-exudative (dry) or exudative (wet). Whereas wet AMD can be treated using anty-VEGF injections with a good clinical result, for the dry form of the disease there is no causative treatment $[2,4,5]$. Thus, the question appears - is it possible to prevent $\mathrm{AMD}$ and/or slow down its progression?

The pathogenesis of the disease is multifactorial and not fully understood but many publications point out the significant role of oxidative stress (OS) $[6,7]$. In the course of AMD, the loss of retinal pigment epithelium (RPE) cells and photoreceptors in the macula area is observed. The retinal examination reveals the presence of drusen - extracellular deposits, which are located between Bruch's membrane and RPE. RPE cells have a high metabolic rate and therefore have the ability to produce reactive oxygen species (ROS). What's more, they are exposed to ROS through iron ion accumulation, exposure to high light and smoking. The pigment epithelium consisting of two carotenoids, which are lutein and zeaxanthin [two stereoisomers: (3R, $\left.3^{\prime} \mathrm{R}\right)$ zeaxanthin, and (3R,3'S)-zeaxanthin (meso-zeaxanthin)], is a natural protective barrier for the retina of the human eye from OS [7]. This discovery became the basis for the use of substances with antioxidant properties such as carotenoids (lutein, zeaxanthin), vitamin $\mathrm{C}$ and $\mathrm{E}$ and zinc in the prevention and treatment of this disease. 
There are several modifiable and non-modifiable risk factors connected with the progression of AMD. The most important are sex, age, genetic, diet, smoking, sunlight exposure, obesity and hypertension $[8,9]$. In this paper, we would like to emphasize the protective role of diet in the disease progression.

A review of the latest and most frequently cited literature was carried out. We searched for the articles which focus on the relationship between nutritional factors and AMD progression. They included works related to the diet in age-related macular degeneration and the latest information on AMD treatment methods.

Scientific research in the field of biochemistry was also cited, in which the level of dietary components in the supportive treatment of AMD was determined.

\section{THE ROLE OF SUPPLEMENTATION IN AMD}

One of the most significant multicentral clinical trials that evaluate the role of antioxidants supplementation on the rate of AMD progression were The Age-Related Eye Disease Study (AREDS) and The Age-Related Eye Disease Study 2 (AREDS2).

The Age-Related Eye Disease Study involved 4757 patients, aged 55 to 80 years, presenting of extensive small drusen, intermediate or large drusen, noncentral geographic atrophy, or pigment abnormalities in one or both eyes, or advanced AMD or vision loss due to AMD in one eye. Patients were randomly assigned to one of four groups depending on the type of oral supplementation (Tab. 1):

- antioxidants (vitamin C - $500 \mathrm{mg}$; vitamin E $-400 \mathrm{IU}$; and $\beta$-carotene $-15 \mathrm{mg}$ );

— zinc - $80 \mathrm{mg}$ (as zinc oxide) and copper $-2 \mathrm{mg}$ (as cupric oxide);

- antioxidants plus zinc;

- placebo [10].

The study revealed that patients receiving zinc, antioxidants, or the combination of both showed a decrease in the rate of progression to advanced AMD. It was estimated that those receiving both zinc and antioxidants achieved a $25 \%$ reduction of progression to advanced AMD relative to placebo. The decreased percentage in visual acuity scores from $29 \%$ for those assigned to placebo to $23 \%$ for those assigned to antioxidants plus zinc. Moreover, the AREDS showed a statistically significant association between the incidence of AMD and risk
Table 1. Commercially available formulas based on the Age-Related Eye Disease Study - AREDS/AREDS2

\begin{tabular}{|l|c|c|}
\hline Nutrient & AREDS formula & AREDS2 formula \\
\hline Vitamin C & $400 \mathrm{mg}$ & $500 \mathrm{mg}$ \\
\hline Vitamin E & $400 \mathrm{IU}$ & $400 \mathrm{IU}$ \\
\hline$\beta$-carotene & $15 \mathrm{mg}$ & - \\
\hline Lutein & - & $10 \mathrm{mg}$ \\
\hline Zeaxanthin & - & $2 \mathrm{mg}$ \\
\hline Zinc & $80 \mathrm{mg}$ & $80 \mathrm{mg}$ \\
\hline Copper & $2 \mathrm{mg}$ & $2 \mathrm{mg}$ \\
\hline
\end{tabular}

factors such as smoking, BMI, race, education, and age [11].

Due to the significant progress in the study of the effect of supplementation on AMD development, soon after the publication of the results of AREDS in 2001, it was necessary to start further research in this direction, that was AREDS2 [11]. Modification of supplementation was also caused by reports of an increased risk of lung cancer in smokers after using $\beta$-carotene as well as urogenital infections resulting from taking zinc $[12,13]$.

In addition, the natural occurrence of lutein and zeaxanthin in the pigment epithelium was the basis for using these substances in further studies on AMD supplementation. The aim of the AREDS2 was to show whether adding lutein + zeaxanthin, omega-3 long-chain polyunsaturated fatty acids [docosahexaenoic acid (DHA) + eicosapentaenoic acid (EPA)], or both to the AREDS formulation reduces the risk of developing advanced AMD. It is worth mentioning that DHA is an important structural component of the retina, and EPA may play the role of a precursor to signalling molecules that can affect retinal function, which was the basis for studying these nutrients. The second aim of the research was to evaluate the effect of eliminating $\beta$-carotene, lowering zinc doses, or both in the AREDS formulation [14]. The conclusion of the AREDS2 was that comparing to placebo, the addition of lutein/zeaxanthin and/or omega-3 fatty acids to the previous AREDS formulation had no significant effect on AMD progression of visual acuity loss. Moreover, the results showed no effect of $\beta$-carotene elimination or lower-dose zinc on progression to advanced AMD. However, because of potential increased incidence of lung cancer in former smokers, lutein + zeaxanthin could be an appropriate carotenoid substitute in the AREDS formulation. Addition of lutein and zeaxanthin may have some 
effect on AMD progression in those populations in which the dietary intake of these nutrients is low.

Over the years, many studies have been conducted to assess the effect of antioxidants supplementation on AMD. Feng et al. based on a meta-analysis of nine randomized trials (a total of 920 eyes) have shown that lutein supplementation at a dose of 10 or $20 \mathrm{mg}$ daily for more than 6 months can contribute to an increase in macular pigment optical density (MPOD), improvement of visual acuity and a better sense of contrast in patients with AMD [15]. Macular pigment levels, usually measured in terms of MPOD, may correspond with normal retinal function [15-20].

The role of omega-3 fatty acids supplementation in AMD is also worth mentioning. They have the ability to renew in RPE cells, their deficiency can lead to degradation of photoreceptors as well as the accumulation of lipid filled drusen in RPE and sub-RPE space [21]. A number of studies have been conducted to assess the effects of omega-3 supplementation on the development and progression of AMD. The Eye Disease Case-Control Study showed that the higher intake of $n-3$ fatty acids may be associated with the lower risk of AMD among patients with the lower linoleic acid (omega-6 fatty acid) consumption $[22,23]$. The Nutritional AMD Treatment 2 (NAT2) study showed that higher EPA and DHA content in red blood cell membranes may have protective effects against AMD [24]. Some authors suggest that high doses of EPA $(3.4 \mathrm{~g})$ and DHA (1.6 g) daily for six months can result in improved visual acuity in patients with dry AMD [25]. On the other hand, a 2015 Cochrane meta-analysis report that there is no evidence confirming that supplementation with Omega-3 fatty acids prevents or slows the progression of AMD [26].

To sum up, although some scientific studies indicate a positive effect of omega- 3 fatty acid supplementation in preventing the progression of AMD, it should be emphasized that most studies are observational and not supported by randomized controlled studies $[21,26]$. Therefore, it seems appropriate to recommend that patients eat fatty fish or omega-3 fatty acid supplements as potentially beneficial in reducing the progression of AMD while informing patients that most of the data is based on observational studies [26].

Noteworthy is also another substance - resveratrol, which is a plant polyphenolic compound commonly found in peel and grape seeds as well as in red and white wine [27]. Resveratrol has antioxidant, anti-angiogenic and anti-inflammatory properties, which are used not only in the prevention of eye diseases but also in the prevention of cardiovascular, cancer and neurodegenerative diseases [28]. Based on the literature, it is known that resveratrol acts on various types of eye cells by increasing the level of natural enzymes and the ability to molecularly defend antioxidants. This substance, by limiting pro-inflammatory factors such as interleukins and prostaglandins, as well as reducing chemical attraction and recruitment of immune cells to the inflammatory site, has anti-inflammatory capabilities. In addition, resveratrol has been shown to have anti-VEGF activity and inhibits vascular endothelial cell proliferation and migration. Resveratrol has been shown to be successfully used in many ophthalmic diseases based on animal models and in vivo experiments [28].

One of the more interesting and recent studies on the effects of resveratrol on RPE cells is the study by Nashine et al. The study evaluated the effects of over-the-counter nutrient supplements containing resveratrol and HPLC-purified resveratrol, in human transmitochondrial age-related macular degeneration (AMD) retinal pigment epithelial (RPE) patient cell lines. These cell lines were obtained from dry and wet AMD patients. It turned out that resveratrol treatment with different resveratrol formulations improved cell viability and decreased reactive oxygen species generation in each AMD patient cell line. Although further studies are required to establish the cytoprotective potential of resveratrol under different physiological conditions, this novel study established the positive effects of resveratrol supplements in macular degeneration patient cell lines in vitro [27]. The study used a maximum concentration of $1000 \mu \mathrm{M}$ resveratrol as this dose was confirmed to be effective in previous studies that evaluated a wide range of resveratrol doses in AMD RPE [27].

\section{DIET IN AMD}

When discussing the role of dietary supplements in the context of AMD, it should be emphasized that any supplementation can replace a proper, well-balanced diet. Therefore, it is important to determine which nutritional ingredients and in what proportions may have a beneficial effect in the prevention of AMD and patients with already diagnosed disease. Many studies have tried to determine the dietary patterns recommended in AMD 
but it still seems difficult to develop general guidelines based on them [29-31]. Koning-Backus et al. investigated whether general dietary guidelines for healthy lifestyles are associated with a reduced risk of AMD. The study analyzed recommended minimum intake values for various food categories and standards, based on publicly available dietary recommendations from national food centers operating in many developed countries. In addition, they calculated the percentage of the population that followed these recommendations, characterized their profile, and examined the risk of AMD.

The study included four thousand two hundred and two participants in the Rotterdam study $\geq 55$ years who were free from AMD at baseline and followed for $9.1 \pm 5.8$ years. Dietary data were collected using a verified 170 food frequency questionnaire, and food consumption was divided into nutritional patterns based on Health Council guidelines.

The results of the study were as follows - seven hundred and fifty-four people developed AMD. The consumption of the recommended amounts of vegetables $(\geq 200 \mathrm{~g} /$ day $)$, fruits $(2 \times /$ day $)$ and fish $(2 \times /$ week $)$ was $30.6 \%, 54.9 \%$ and $12.5 \%$ respectively. In particular, fish consumption $(2 \times /$ week $)$ reduced the risk of AMD. Consumption of the recommended amounts of all 3 food groups was only $3.7 \%$, but following this pattern showed a further reduction in the risk of AMD.

To sum up, a diet of $200 \mathrm{~g}$ a day of vegetables, fruit twice a day and fish twice a week is associated with a significantly reduced risk of AMD [32].

A systematic literature review conducted by Chapman et al. identified eighteen high-quality studies evaluating the role of diet and food intake in AMD. This work showed that the diet has a multifactorial effect on the occurrence and progression of AMD. The authors of the review recommend adhering to the Mediterranean diet, which is based on a high intake of fruits, vegetables, legumes, whole grains and nuts; moderate consumption of fish, poultry and dairy products, the use of olive oil instead of fats, small amounts of red wine and limited consumption of red meat. As for other diets, the Oriental diet pattern with higher intake of vegetables, legumes, fruits, whole grains, tomatoes and seafood is considered more favorable compared to the Western diet pattern, which is characterized by higher consumption of red meat, processed meat, and high-fat products dairy fried potatoes, refined beans and eggs. In addition, it was noted that high consumption of vegetables rich in carotenoids and fatty fish containing omega- 3 fatty acids were beneficial for those at risk of AMD. However, in order to reduce the risk of AMD progression, a minimum intake of vegetable oils and animal fats containing omega- 6 fatty acids and red or processed meat is recommended. In addition, foods with a low glycemic index (IG) are preferred to foods with a high IG, and alcohol consumption should be limited.

\section{CONCLUSION}

The treatment of the dry form of age-related macular degeneration has been a big challenge for scientists for many years. To date, despite numerous scientific studies including clinical trials, no causative treatment of this disease is known. Among the modifiable factors affecting the incidence and progression of AMD, it has been shown that supplementation and, above all, a proper diet are very important. The currently recommended dietary supplementation in AMD patients has been developed based on the AREDS and AREDS2 studies (Tab. 2). This formula includes the intake of $500 \mathrm{mg}$ vitamin C, 400 IU. vitamin E, $25 \mathrm{mg}$ zinc, $2 \mathrm{mg}$ copper, $10 \mathrm{mg}$ lutein and $2 \mathrm{mg}$ zeaxanthin per day. A special role is assigned to xanthophyll carotenoids-lutein and zeaxanthin, which are the main components of the macular pigment. They are thought to play an important role in maintaining the morphological and functional integrity of the retina. Concentrated in the macula, these substances have the ability to filter blue light and

Table 2. Recommended composition of dietary
supplements for patients with age-related macular
degeneration (AMD) and their sources in nutritional
products


deactivate reactive oxygen species, thus protecting against light-induced oxidative damage that is involved in AMD pathogenesis. Studies have shown that consumption of lutein and zeaxanthin is associated with a lower risk of AMD progression, but remaining inconsistent about preventive effects of supplementation on AMD.

As mentioned earlier, diet has a vital role in AMD, so the provision of appropriate nutritional advice to those at risk of AMD is recommended. Current evidence suggests that improving diet quality, increasing the intake of foods containing the nutrients required by the retina, and avoiding foods that cause oxidative damage will play an important role in protecting against AMD. With the approaching increase in the number of AMD patients in the aging population, healthcare professionals should actively promote food choices that minimize AMD risk.

\section{Acknowledgements}

Foundation for Polish Science is acknowledged for financial support within the project "Xanthophylls in the Retina of the Eye (TEAM/2016-3/21).

\section{REFERENCES}

1. Bird AC, Bressler NM, Bressler SB, et al. An international classification and grading system for age-related maculopathy and agerelated macular degeneration. Surv Ophthalmol. 1995; 39(5): 367-374, doi: 10.1016/s0039-6257(05)80092-x.

2. Wong WL, Su X, Li X, et al. Global prevalence of age-related macular degeneration and disease burden projection for 2020 and 2040: a systematic review and meta-analysis. Lancet Glob Health. 2014; 2(2): e106-e116, doi: 10.1016/S2214-109X(13)70145-1, indexed in Pubmed: 25104651

3. Nowak JZ. AMD - the retinal disease with an unprecised etiopathogenesis: in search of effective therapeutics. Acta Pol Pharm . 2014; 71(6): 900-916.

4. Pennington KL, DeAngelis MM. Epidemiology of age-related macular degeneration (AMD): associations with cardiovascular disease phenotypes and lipid factors. Eye Vis (Lond). 2016; 3: 34, doi: 10.1186/ s40662-016-0063-5, indexed in Pubmed: 28032115.

5. Rinninella E, Mele MC, Merendino N, et al. The Role of Diet, Micronutrients and the Gut Microbiota in Age-Related Macular Degeneration: New Perspectives from the Gut Retina Axis. Nutrients. 2018; 10(11), doi: 10.3390/nu10111677, indexed in Pubmed: 30400586.

6. Datta S, Cano M, Ebrahimi K, et al. The impact of oxidative stress and inflammation on RPE degeneration in non-neovascular AMD. Prog Retin Eye Res. 2017; 60: 201-218, doi: 10.1016/j.preteyeres.2017.03.002, indexed in Pubmed: 28336424.

7. Domènech EB, Marfany G. The Relevance of Oxidative Stress in the Pathogenesis and Therapy of Retinal Dystrophies. Antioxidants (Basel). 2020; 9(4), doi: 10.3390/antiox9040347, indexed in Pubmed: 32340220 .

8. Heesterbeek TJ, Lorés-Motta L, Hoyng CB, et al. Risk factors for progression of age-related macular degeneration. Ophthalmic Physiol Opt. 2020; 40(2): 140-170, doi: 10.1111/opo.12675, indexed in Pubmed: 32100327.

9. Shim SH, Kim SG, Bae JH, et al. Risk Factors for Progression of Early Age-Related Macular Degeneration in Koreans. Ophthalmic Epidemiol.
2016; 23(2): 80-87, doi: 10.3109/09286586.2015.1129425, indexed in Pubmed: 26950426.

10. Age-Related Eye Disease Study Research Group. A randomized, placebo-controlled, clinical trial of high-dose supplementation with vitamins $C$ and $E$, beta carotene, and zinc for age-related macular degeneration and vision loss: AREDS report no. 8. Arch Ophthalmol. 2001; 119(10): 1417-1436, doi: 10.1001/archopht.119.10.1417, indexed in Pubmed: 11594942.

11. Gorusupudi A, Nelson K, Bernstein PS. The Age-Related Eye Disease 2 Study: Micronutrients in the Treatment of Macular Degeneration. Adv Nutr. 2017; 8(1): 40-53, doi: 10.3945/an.116.013177, indexed in Pubmed: 28096126.

12. Alpha-Tocopherol, Beta Carotene Cancer Prevention Study Group. The Effect of Vitamin $\mathrm{E}$ and Beta Carotene on the Incidence of Lung Cancer and Other Cancers in Male Smokers. New Engl J Med. 1994; 330(15): 1029-1035, doi: 10.1056/nejm199404143301501, indexed in Pubmed: 8127329.

13. Johnson AR, Munoz A, Gottlieb JL, et al. High dose zinc increases hospital admissions due to genitourinary complications. J Urol. 2007; 177(2): 639-643, doi: 10.1016/j.juro.2006.09.047, indexed in Pubmed: 17222649.

14. Age-Related Eye Disease Study 2 Research Group. Lutein + zeaxanthin and omega-3 fatty acids for age-related macular degeneration: the Age-Related Eye Disease Study 2 (AREDS2) randomized clinical trial. JAMA. 2013; 309(19): 2005-2015, doi: 10.1001/jama.2013.4997, indexed in Pubmed: 23644932.

15. Feng $\mathrm{L}$, Nie K, Jiang $\mathrm{H}$, et al. Effects of lutein supplementation in agerelated macular degeneration. PLoS One. 2019; 14(12): e0227048, doi: 10.1371/journal.pone.0227048, indexed in Pubmed: 31887124.

16. Bernstein PS, Li B, Vachali PP, et al. Lutein, zeaxanthin, and mesozeaxanthin: The basic and clinical science underlying carotenoid-based nutritional interventions against ocular disease. Prog Retin Eye Res. 2016; 50: 34-66, doi: 10.1016/j.preteyeres.2015.10.003, indexed in Pubmed: 26541886.

17. Ozawa $Y$, Shigeno $\mathrm{Y}$, Nagai $\mathrm{N}$, et al. Absolute and estimated values of macular pigment optical density in young and aged Asian participants with or without age-related macular degeneration. BMC Ophthalmol. 2017; 17(1): 161, doi: 10.1186/s12886-017-0557-5, indexed in Pubmed: 28851319.

18. Loane E, Kelliher C, Beatty $S$, et al. The rationale and evidence base for a protective role of macular pigment in age-related maculopathy. $\mathrm{Br} \mathrm{J}$ Ophthalmol. 2008; 92(9): 1163-1168, doi: 10.1136/bjo.2007.135566, indexed in Pubmed: 18669545.

19. Sabour-Pickett S, Nolan JM, Loughman J, et al. A review of the evidence germane to the putative protective role of the macular carotenoids for age-related macular degeneration. Mol Nutr Food Res. 2012; 56(2): 270-286, doi: 10.1002/mnfr.201100219, indexed in Pubmed: 22121091.

20. Puell MC, Palomo-Alvarez C, Barrio AR, et al. Relationship between macular pigment and visual acuity in eyes with early age-related macular degeneration. Acta Ophthalmol. 2013; 91(4): e298-e303, doi: 10.1111/aos.12067, indexed in Pubmed: 23575039

21. Mukhtar S, Ambati BK. The value of nutritional supplements in treating Age-Related Macular Degeneration: a review of the literature. Int Ophthalmol. 2019; 39(12): 2975-2983, doi: 10.1007/s10792-01901140-6, indexed in Pubmed: 31313070.

22. Aoki A, Inoue M, Nguyen E, et al. Dietary n-3 Fatty Acid, -Tocopherol, Zinc, vitamin D, vitamin $C$, and $\beta$-carotene are Associated with AgeRelated Macular Degeneration in Japan. Sci Rep. 2016; 6: 20723, doi: 10.1038/srep20723, indexed in Pubmed: 26846575.

23. Seddon JM, George S, Rosner B. Cigarette smoking, fish consumption, omega-3 fatty acid intake, and associations with age-related macular degeneration: the US Twin Study of Age-Related Macular Degeneration. Arch Ophthalmol. 2006; 124(7): 995-1001, doi: 10.1001/ archopht.124.7.995, indexed in Pubmed: 16832023.

24. Borrelli E, Souied EH, Freund KB, et al. The role of omega-3 and micronutrients in age-related macular degeneration. Surv Ophthalmol. 2014; 59(5): 532-539, doi: 10.1016/j.survophthal.2014.01.001, indexed in Pubmed: 24657038. 
25. Georgiou T, Neokleous A, Nicolaou D, et al. Pilot study for treating dry age-related macular degeneration (AMD) with high-dose omega-3 fatty acids. PharmaNutrition. 2014; 2(1): 8-11, doi: 10.1016/j. phanu.2013.10.001.

26. Lawrenson JG, Evans JR. Omega 3 fatty acids for preventing or slowing the progression of age-related macular degeneration. Cochrane Database Syst Rev. 2012; 11: CD010015, doi: 10.1002/14651858. CD010015.pub2, indexed in Pubmed: 23152282.

27. Nashine $S$, Nesburn AB, Kuppermann BD, et al. Role of Resveratrol in Transmitochondrial AMD RPE Cells. Nutrients. 2020; 12(1), doi: 10.3390/nu12010159, indexed in Pubmed: 31935891.

28. Lançon A, Frazzi R, Latruffe N. Anti-Oxidant, Anti-Inflammatory and Anti-Angiogenic Properties of Resveratrol in Ocular Diseases. Molecules. 2016; 21(3): 304, doi: 10.3390/molecules21030304, indexed in Pubmed: 26950104.

29. Amirul Islam FM, Chong EW, Hodge AM, et al. Dietary patterns and their associations with age-related macular degeneration: the Melbourne collaborative cohort study. Ophthalmology. 2014; 121(7): 1428-1434.e2, doi: 10.1016/j.ophtha.2014.01.002, indexed in Pubmed: 24560564.

30. Chiu CJ, Chang ML, Zhang FF, et al. The relationship of major American dietary patterns to age-related macular degeneration. Am J Ophthalmol. 2014; 158(1): 118-127.e1, doi: 10.1016/j.ajo.2014.04.016, indexed in Pubmed: 24792100.

31. Merle B, Silver B, Rosner JM. Adherence to a Mediterranean diet, genetic susceptibility, and progression to advanced macular degeneration: a prospective cohort study. Am J Clin Nutr. 2015; 102(5): $1196-$ 1206, doi: 10.3945/ajcn.115.111047, indexed in Pubmed: 26490493.

32. de Koning-Backus APM, Buitendijk GHS, Kiefte-de Jong JC, et al. Intake of Vegetables, Fruit, and Fish is Beneficial for Age-Related Macular Degeneration. Am J Ophthalmol. 2019; 198: 70-79, doi: 10.1016/j. ajo.2018.09.036, indexed in Pubmed: 30312575.

33. Chapman NA, Jacobs RJ, Braakhuis AJ. Role of diet and food intake in age-related macular degeneration: a systematic review. Clin Exp Ophthalmol. 2019; 47(1): 106-127, doi: 10.1111/ceo.13343, indexed in Pubmed: 29927057. 